\title{
El efecto de la calidad del servicio en la satisfacción del derechohabiente en instituciones públicas de salud en México
}

\author{
Assessing the effect of service quality over user satisfaction in public health \\ institutions in Mexico
}

\author{
Jorge Vera* y Andrea Trujillo \\ Tecnológico de Monterrey, EGADE Business School, México
}

Recibido el 6 de mayo del 2015; aceptado el 13 de julio del 2015

Disponible en Internet el 19 de marzo de 2018

\section{Resumen}

El objetivo de este trabajo es ofrecer una explicación de los factores que tienen impacto en la percepción de satisfacción del derechohabiente del sistema público de salud. Mediante un estudio exploratorio y analizando el proceso de atención que recibe un derechohabiente en una clínica de primer nivel, se detectaron 32 atributos de calidad en el servicio. Se diseñó un cuestionario estructurado para medir la percepción sobre el cumplimiento de estos atributos, así como indicadores de satisfacción. Se integró una muestra de 600 derechohabientes dividida de forma equitativa entre tres instituciones: Secretaría de Salud, Instituto Mexicano del Seguro Social e Instituto de Seguridad y Servicios Sociales de los Trabajadores del Estado. Los atributos se integraron en siete dimensiones de calidad del servicio mediante un análisis factorial confirmatorio. Para analizar el impacto de estas dimensiones en la satisfacción se llevaron a cabo tanto seis modelos de regresión como dos modelos estructurales. Estos análisis arrojaron que la calidad del servicio proporcionado por los médicos, así como la calidad del servicio de la entrega de medicamentos, tienden a ser los factores de mayor impacto en la satisfacción del derechohabiente. Calidad del servicio del personal de análisis clínicos y la calidad de las instalaciones también tuvieron impactos significativos. Sin embargo, factores como calidad del servicio de los enfermeros(as), calidad del servicio del personal de farmacia, y calidad en la recepción (turnos), no tuvieron un impacto estadísticamente significativo.

\footnotetext{
*Autor para correspondencia.

Correo electrónico: jorge.vera@itesm.mx (J. Vera).

La revisión por pares es responsabilidad de la Universidad Nacional Autónoma de México.
} 
Códigos JEL: M31, M39, I11, I18

Palabras clave: calidad en el servicio, satisfacción, instituciones de salud

\begin{abstract}
This work aims to offer an explanation of the service quality components that have an effect on health public institutions user satisfaction. The study focuses only on first level clinics. First, through an exploratory examination, in which the attention process was analyzed, 32 service quality attributes were detected. A structured questionnaire was designed in order to measure perceived attribute performance and satisfaction, as well as to conduct interviews with users. A sample of $n=600$ users of three different health public institutions were conformed. To assess the effect of service quality attributes over user's satisfaction, two types of analysis were carried out: six regression models and two structural models are presented. Medicine delivery perceived quality and doctor service perceived quality were the latent variables with the higher effect over user satisfaction. Other latent variables were also significant, such as: clinical analyses perceived quality and facilities perceived quality. Latent variables such as nurse service perceived quality and pharmacy staff service perceived quality did not have a significant effect over satisfaction.
\end{abstract}

JEL Classification: M31, M39, I11, I18

Key words: service quality, customer satisfaction, health care institutions

\title{
Introducción
}

La medición de calidad del servicio ha sido un tema largamente abordado en la literatura de investigación en el área de marketing. Aunque el contexto usual en que es utilizado se refiere a la evaluación del servicio en negocios de servicios y de productos que involucran alguna forma de servicio en la atención al cliente, también es utilizable en el contexto de servicios que el Estado y el gobierno presta a la población en general como se puede ver en: Scott y Shieff (1993), Donnelly et al. (1995), Andaleeb (2001), y en Olin et al. (2014), sólo por mencionar a algunos.

La calidad del servicio ha sido establecida anteriormente como un grupo de factores que tiende a tener un efecto importante en la satisfacción y en la retención de clientes y usuarios (Taylor y Baker, 1994; Spreng y Mackoy, 1996; Zeithaml et al., 1996). Así, se ha documentado ampliamente que cuando un usuario percibe que se cumple adecuadamente los atributos ofrecidos, esto ayuda a generar en ellos la percepción del cumplimiento de sus expectativas, así como un sentimiento general de satisfacción. De esta forma, el presente trabajo se enmarca de forma teórica en la relación entre percepción de calidad del servicio y la satisfacción del usuario (cliente).

De acuerdo a Gomez et al. (2011) el sistema mexicano de salud se conforma por dos grandes sectores: público y privado. A su vez el sector público está formado por dos tipos de instituciones. Por un lado, las instituciones de seguridad social a los que los empleados de empresas establecidas tienen derecho, y por otro, las organizaciones y programas que atienden a la población que no goza de seguridad social. Dentro del grupo de seguridad social están el 
Instituto Mexicano del Seguro Social (IMSS) que atiende aproximadamente al $80 \%$ de este tipo de derechohabientes, el Instituto de Seguridad y Servicios Sociales de los Trabajadores del Estado (ISSSTE), Petróleos Mexicanos (PEMEX), Secretaría de la Defensa (SEDENA), Secretaría de Marina (SEMAR), entre otros. En el grupo de organismos para población que no goza de seguridad social (personas que no cuentan con actividad económica "reconocida") están la Secretaría de Salud (SSa), los servicios estatales de salud (SESA), el programa IMSSOportunidades (IMSS-O), y el Seguro Popular de Salud (SPS).

El presente estudio pretendió enfocarse en clínicas de primer nivel ${ }^{2}$ de las tres principales instituciones públicas de salud: IMSS e ISSSTE del subsector de seguridad social y SSa que pertenece al grupo que atiende a la población que no goza de seguridad social. A partir de esto, este estudio ofrece un acercamiento a la identificación de los atributos de calidad del servicio en el proceso de atención al derechohabiente en este tipo de instituciones del Estado. De esta forma, el propósito de este trabajo apunta a determinar y explicar el efecto de las dimensiones de calidad del servicio de este tipo de instituciones en la satisfacción del derechohabiente. Así mismo, se pretende discriminar qué dimensiones son las de mayor influencia en esta satisfacción. Con esto, se abona a la comprensión de la satisfacción del usuario de este tipo de servicios, así como a la identificación de qué aspectos del servicio pueden ser mejorados para aumentar la percepción de satisfacción por parte de los derechohabientes.

\section{Calidad en el servicio}

Para poder mejorar la calidad en un servicio es necesario tener claro qué es y cómo medirla. La calidad en el servicio tiende a ser más difícil de establecer y medir que la calidad de un producto (Parasuraman, Zeithaml y Berry, 1985), esto debido principalmente a la intangibilidad y heterogeneidad de los servicios. La calidad del servicio comenzó a recibir mayor atención a partir de la década de los ochenta, fue ahí cuando algunos de los autores seminales sobre calidad del servicio propusieron definiciones y comenzaron a proponer formas para su medición (Oliver, 1980; Parasuraman et al., 1985, Gronroos, 1984). Una tendencia generalizada es que la calidad del servicio no se mide con respecto a los estándares establecidos por la empresa, sino a partir de la perspectiva (percepción) de los clientes o usuarios (Asubonteng, Mc Vleary y Swan, 1996).

Una de las definiciones más utilizadas en la literatura es la de Parasuraman et al. (1988, p. 16) quienes plantean que la calidad en el servicio es "una sentencia o actitud en relación con la excelencia en general o la superioridad del servicio global". En concordancia, Dagger et al. (2007) proponen que la medición de calidad del servicio en organizaciones de salud podía hacerse utilizando cuatro enunciados: la calidad general del servicio prestado por la clínica es excelente; la calidad del servicio prestado en la clínica es impresionante; el servicio ofrecido por la clínica es de un alto nivel; la clínica ofrece un servicio que es superior en todos los sentidos.

\footnotetext{
${ }^{2}$ Primer nivel es el más cercano y de primer contacto a la población; consiste en recursos que permiten resolver las necesidades de atención básicas más frecuentes; pueden ser actividades de promoción de salud, prevención y procedimientos de recuperación y rehabilitación; es la puerta de entrada al sistema de salud; se caracteriza por establecimientos de baja complejidad donde se resuelven la mayoría de los problemas prevalentes (Vignolo et al., 2011).
} 


\section{Medición de la calidad del servicio}

Para su medición, la calidad del servicio se ha planteado como un constructo multidimensional, es decir, los clientes no sólo evalúan el resultado que obtienen de una empresa, sino que integran en su evaluación una serie de dimensiones que perciben durante todo el proceso de la entrega del servicio (Parasuraman et al., 1985). El primer instrumento propuesto en la literatura para medir calidad del servicio de forma generalizada es el SERVQUAL, diseñado por Parasuraman, et al. (1988). En este instrumento se propuso la medición del cumplimiento y de la importancia de 22 atributos agrupados en cinco dimensiones: tangibles, aseguramiento, respuesta, empatía y confianza. Asubonteng et al. (1996) reportan numerosas aplicaciones del SERVQUAL en servicios de salud a menos de diez años de haber sido publicado, sin embargo, éste ha recibido considerables críticas sobre qué tan adecuado es utilizar este mismo instrumento en todos los tipos distintos de servicios. Es decir, algunos autores afirman que no todos los servicios tienen las mismas características básicas por lo que se han desarrollado instrumentos específicos enfocados a diferentes industrias (Por citar ejemplos ver: Babakus y Boller, 1992; Van Dyke, et al., 1997; Jabnoun y Khalifa, 2005; Akbaba, 2006; Caro y García, 2007).

Taylor y Cronin (1994) señalan que en la mercadotecnia de servicios de salud se debe tener mucho cuidado en aplicar escalas generales usadas para otros servicios, como el SERVQUAL y el SERVPERF, porque existe un riesgo alto de que se traduzcan equivocadamente al contexto del cuidado de la salud. En la literatura se reportan estudios que han adaptado el SERVQUAL a este sector (Taylor y Cronin, 1994; Kesuma, et al., 2013) pero también hay autores, que, dentro del sector de salud, proponen dimensiones específicas (Woodside, et al., 1989; Hill y McCrory, 1997; Dagger et al., 2007; Lis et al., 2011; Haque, et al., 2012; Kumaraswamy, 2012; Amin y Siti, 2013). Estas dimensiones específicas de calidad del servicio para centros de salud que han sido propuestas anteriormente se resumen en la Tabla 1.

En el presente estudio la medición de calidad en el servicio se realizó siguiendo la propuesta de Cronin y Taylor (1994), en la que señalan que es más apropiado medir la calidad en el servicio preguntando únicamente sobre el desempeño de cada atributo que incorporando adicionalmente la medición de las expectativas del cliente hacia cada atributo como lo propone Parasuraman et al. (1988). Según Parasuraman et al. (1988) la calidad del servicio debe ser la discrepancia entre el desempeño del atributo y las expectativas que se tenían del mismo. Sin embargo, Cronin y Taylor (1994) encuentran evidencias que esta forma de medición genera problemas de estabilidad y confiabilidad, y encuentran mejores indicadores de medición al medir únicamente el desempeño.

\section{Efecto de la calidad del servicio en variables dependientes}

Como ya se ha mencionado, la calidad en el servicio ha sido planteada como antecedente de la satisfacción del cliente y de la lealtad que se puede tener hacia una marca u organización (Knutson et. al., 1991; Taylor y Baker, 1994; Stevens et. al., 1995; Spreng y Mackoy 1996; Dabholkar et. al., 1996; Zeithaml et al., 1996; Chi-Cui et. al., 2003; Gounaris, 2005; Tsoukatos y Rand, 2007; Ryu y Han, 2010; Ryu et al., 2012; Orel y Kara, 2014; Bruwer, 2014; Bansal y Taylor, 2015; De Oña et al., 2015). El caso de los centros de salud no es la excepción; en los últimos años se han realizado estudios para corroborar empíricamente la relación de la calidad en el servicio con la satisfacción y con la lealtad del usuario en este sector (Babakus y Mangold, 
1992; Taylor y Baker, 1994; Atta, 2012; Haque et al., 2012; Kumaraswamy, 2012; Amin y Siti, 2013; Kesuma et al., 2013).

A menudo los conceptos de calidad en el servicio y satisfacción se confunden o se traslapan. En el ámbito de los servicios de salud Woodside et al., (1989) plantearon la satisfacción del paciente como una forma de actitud, ya que es el reflejo de lo mucho que a un paciente le gusta o le disgusta el servicio después de haber tenido la experiencia en el hospital. Ellos identificaron que la satisfacción se da en función de la calidad percibida del servicio. No obstante, calidad del servicio y la satisfacción se comprenden como constructos diferentes. John (1992) explica la diferencia entre ambos constructos señalando que la satisfacción se sugiere medir con una sola dimensión general, mientras que la calidad en el servicio debería medirse con una escala como el SERVQUAL que contempla la partición del servicio en muchos atributos. En el sector de servicios de salud, al determinar el efecto de la calidad del servicio en la lealtad o apego del usuario hacia la institución, la lealtad ha tendido a ser medida en la mayoría de los casos como la intención de retornar a la institución como un indicador aceptable (Dagger, et al., 2007; Amin y Siti, 2013), y no necesariamente como la acción observada de retorno.

La Tabla 1 muestra en orden cronológico dimensiones de calidad del servicio que han sido propuestas anteriormente en estudios que han abordado la medición de calidad del servicio en instituciones de cuidado de la salud. En esta tabla, en la primera columna se reportan los autores y la fecha de publicación, en la segunda se enlistan las dimensiones que se han propuesto, y en la tercera se muestran las variables dependientes de comportamiento en las que tendría efecto las dimensiones de calidad del servicio.

Tabla 1

Estudios que abordan la calidad del servicio en instituciones de salud y su impacto en el comportamiento del usuario

\begin{tabular}{|c|c|c|}
\hline Autor & Dimensiones de calidad del servicio & Variable dependiente \\
\hline Woodside, et al., 1989 & $\begin{array}{l}\text { Admisión. Cuidado de enfermería. } \\
\text { Servicio de comida. Limpieza y mantenimiento. } \\
\text { Servicio técnico. Salida (alta) del hospital. }\end{array}$ & $\begin{array}{l}\text { Satisfacción } \\
\text { Intención de retornar }\end{array}$ \\
\hline Taylor y Cronin, 1994 & Dimensiones del SERVQUAL adaptadas a hospitales. & Satisfacción \\
\hline Hill y McCroy, 1997 & $\begin{array}{l}\text { Factores clínicos: Seguridad. Higiene. Comunicación. } \\
\text { Alivio del dolor. } \\
\text { Factores no clínicos: Disponibilidad de baños. Confort de } \\
\text { las camas. Calidad de las sábanas. Disponibilidad de teléfonos públicos. Calidad } \\
\text { de la comida. }\end{array}$ & $\mathrm{n} / \mathrm{a}$ \\
\hline Dagger et al., (2007) & $\begin{array}{l}\text { Calidad interpersonal: Interacción. Relaciones. } \\
\text { Calidad técnica: Resultados. Experiencia. } \\
\text { Calidad ambiental: Atmósfera. Tangibles. } \\
\text { Calidad administrativa: Momento oportuno. Operación. Soporte. }\end{array}$ & $\begin{array}{l}\text { Satisfacción } \\
\text { Intención de retornar }\end{array}$ \\
\hline Lis, et al., (2011) & $\begin{array}{l}\text { Operaciones y servicios. } \\
\text { Equipo multidisciplinario para el cuidado del paciente. }\end{array}$ & Recomendación a otros \\
\hline Atta (2012) & Dimensiones del SERVQUAL adaptadas a hospitales. & Satisfacción \\
\hline Haque et al., (2012) & Personal de apoyo. Instalaciones del hospital. Atención al cliente. & Satisfacción \\
\hline Kumaraswamy (2012) & Conducta del médico. Personal de Apoyo. Atmósfera. Desempeño operativo. & Satisfacción \\
\hline Amin y Siti (2013) & Admisión. Servicio Médico. Servicio general. Alta médica. Responsabilidad social. & $\begin{array}{l}\text { Satisfacción } \\
\text { Intención de retornar }\end{array}$ \\
\hline Kesuma et al., (2013) & Dimensiones del SERVQUAL adaptadas a hospitales. & $\begin{array}{l}\text { Lealtad } \\
\text { CRM }\end{array}$ \\
\hline
\end{tabular}


Si se observa la Tabla 1 se puede ver que las dimensiones de calidad en el servicio que han sido utilizadas anteriormente varían de forma importante con las dimensiones de calidad del servicio que son utilizadas en este estudio (como se verá más adelante). Esto se debe a que la gran mayoría de los estudios previos se centran en la calidad del servicio de centros de salud privados y sus procesos de atención a usuarios varían considerablemente con respecto al proceso de atención que se utiliza en las instituciones públicas de salud. Uno de los elementos novedosos que aporta este estudio es que se ha realizado precisamente en este último tipo de instituciones.

\section{Planteamiento}

De forma preliminar se llevó a cabo un estudio exploratorio con el propósito de detectar los atributos relevantes de calidad en el servicio para derechohabientes del sector público de salud en clínicas de primer nivel. Como parte de este estudio preliminar, se revisó el proceso completo por el que pasa un derechohabiente en un día normal en que acude a consulta médica. A partir de este proceso se indagó en cuáles son los atributos que los derechohabientes esperaban que fueran cumplidos. De esta forma se detectó que en términos generales en las tres instituciones cubiertas en este trabajo (IMSS, ISSSTE, SSa) la atención contempla los siguientes siete elementos separados por tiempos de espera: recepción y solicitud de turno (ficha), contacto inicial con enfermera(o) para toma de datos, atención del médico, atención del personal de la farmacia, aspectos de la calidad en la entrega de los medicamentos, atención del personal de análisis clínicos, y las instalaciones en general. Para cada uno de estos aspectos se detectaron atributos específicos de calidad en el servicio mediante la realización de treinta y seis entrevistas a profundidad con derechohabientes que acuden con regularidad a recibir atención médica a estas instituciones. Estos atributos se muestran en la Tabla 2 junto con los indicadores de calidad del servicio que fueron incorporados.

A partir de lo anterior, se conjeturó que estos aspectos de la calidad del servicio debían tener un efecto positivo directo sobre la satisfacción del derechohabiente. Como ya fue mencionado se ha establecido anteriormente que la calidad en el servicio puede ser un antecedente importante para la satisfacción del usuario (Knutson et. al., 1991; Taylor y Baker, 1994; Stevens et. al., 1995; Spreng y Mackoy 1996; Dabholkar et. al., 1996; Zeithaml et al., 1996; Chi-Cui et. al., 2003; Gounaris, 2005; Tsoukatos y Rand, 2007; Ryu y Han, 2010; Ryu et al., 2012; Orel y Kara, 2014; Bruwer, 2014; Bansal y Taylor, 2015; de Oña et al., 2015). Así mismo, se ha probado la relación entre calidad del servicio como un antecedente de la satisfacción del paciente concretamente en servicios de salud (Babakus y Mangold, 1992; Taylor y Baker, 1994; Atta, 2012; Haque et al., 2012; Kumaraswamy, 2012; Amin y Siti, 2013; Kesuma et al., 2013), aunque no específicamente en clínicas de primer nivel de instituciones del Estado (o gubernamentales). De esta forma se plantearon siete hipótesis, donde cada una se refiere a que se espera que exista un efecto positivo entre cada uno de los siete aspectos (posibles dimensiones) de la calidad del servicio hacia la satisfacción del derechohabiente (usuario). Estas siete hipótesis se esquematizan en la Figura 1. 


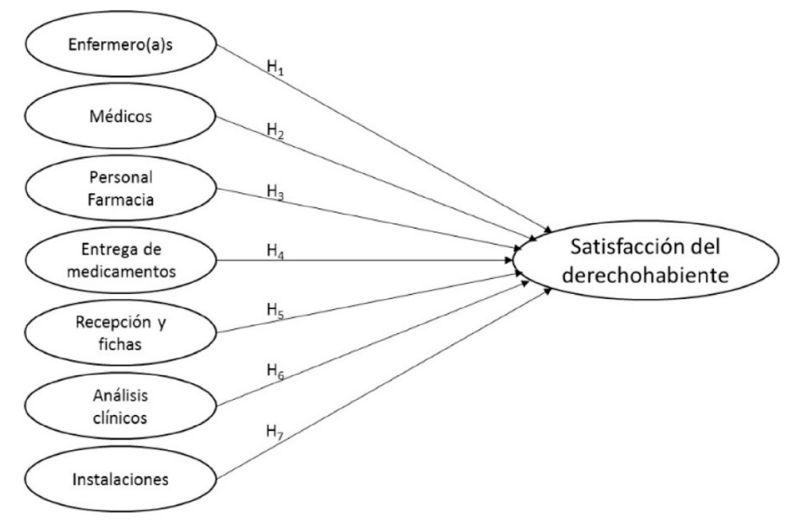

Figura 1. Planteamiento e hipótesis

Aunque en este tipo de estudios típicamente se plantea la satisfacción como un factor mediador entre la calidad del servicio y la lealtad del cliente, en este caso la relación sólo se plantea entre calidad del servicio (como variables independientes) y la satisfacción como variable dependiente final. Esto debido a que en el sector de instituciones de salud del Estado, el derechohabiente tiene pocas posibilidades de elegir la institución en la cual atender sus necesidades de salud. Aquí, un trabajador del Estado es forzosamente inscrito en el ISSSTE y un trabajador del sector privado es forzosamente inscrito al IMSS. Así, aquel mexicano que no goza de seguridad social, le queda como única posibilidad, por parte del Estado, la SSa o el Seguro Popular. Por esta razón no se implementan variables relacionadas a las preferencias e intenciones del cliente. Sin embargo, aunque el derechohabiente tiene pocas posibilidades de elección, aquí se considera que debe ser obligación del Estado, y de estas instituciones, ofrecer el mejor servicio posible a sus afiliados (clientes).

\section{Metodología}

Dado que en el propósito del estudio se pretendió evaluar el servicio que de hecho se está ofreciendo a los derechohabientes, se llevó a cabo un estudio con un diseño causal de tipo no experimental post-hoc (Kerlinger, 1979). Para esto, se realizaron entrevistas estructuradas mediante cuestionario. Como ya se mencionó anteriormente, para el diseño del cuestionario se llevaron a cabo treinta y seis entrevistas a profundidad con derechohabientes. Estas entrevistas tuvieron la finalidad de identificar atributos relevantes de calidad en el servicio que las personas esperan que se cumplan en la atención que reciben por parte de la clínica de primer nivel a la que regularmente asisten. Se identificaron treinta y dos atributos en total. La lista de estos atributos detectados puede verse en la Tabla 2. Los reactivos utilizados para medir el cumplimiento de cada uno de estos atributos, tal y como aparecen en el cuestionario, pueden verse en la Tabla 3. Estos reactivos fueron asociados a escalas de Likert de seis categorías de respuesta (excelente, muy buena, buena, mala, muy mala, pésima). El cuestionario se diseñó para ser aplicado en una entrevista persona a persona utilizando apoyos gráficos para mostrar al respondiente las categorías de respuesta de los reactivos. 
Se conformó una muestra de $n=600$ derechohabientes seleccionados por conveniencia y divididos de forma equitativa entre las tres instituciones utilizadas en el presente estudio (IMSS, ISSSTE y SSa). Las clínicas donde se realizaron las entrevistas fueron elegidas procurando tener una cobertura territorial sobre toda la Ciudad de México. Las entrevistas fueron realizadas en clínicas de primer nivel de estas tres instituciones. Como se solicitaba que evaluaran el servicio completo recibido el día en cuestión, los entrevistadores debían solicitar a los derechohabientes responder a la entrevista una vez terminado el proceso de atención (recepción-turno-datos/enfermera-espera-médico-espera-entrega/medicamentos). Los derechohabientes que respondieron fueron seleccionados por intercepción dentro, o al salir, de la clínica. Las clínicas que fueron seleccionadas para realizar las entrevistas se distribuyen en diferentes zonas de la Ciudad de México. Se procuró elegir clínicas dentro de cada una de las delegaciones políticas (municipios) que conforman esta ciudad.

\section{Análisis preliminar de datos}

Para el análisis de datos, primero se llevó a cabo análisis factorial confirmatorio para corroborar una separación coherente de los atributos incluyendo las mediciones de satisfacción. De esta forma se generaron siete componentes (dimensiones) de calidad en el servicio y una dimensión de satisfacción que posteriormente hizo la función de variable dependiente latente en el análisis estructural. La integración de estos componentes producto de un análisis factorial puede verse en la Tabla 2. Esta separación coherente de atributos tiende a dar evidencia de la valides de medición de los reactivos y de las dimensiones (variables latentes) resultantes.

Para este análisis factorial, y para lograr la separación de componentes, se utilizó una rotación oblicua que no asegura ortogonalidad (correlación cero entre componentes). Se optó por este método de rotación debido a que el componente satisfacción debía ser utilizado posteriormente como variable dependiente por lo que no se quería una correlación cero con los demás componentes. De cualquier forma el análisis estructural soporta bien, y en muchos casos se asume, cierta correlación entre variables independientes latentes como un fenómeno común. Así, al interpretar el contenido de los componentes con respecto a las cargas factoriales de cada uno de los atributos, se determinó que los componentes obtenidos son los siguientes:
a) Calidad del servicio proporcionado por las/los enfermera(o)s $\left(\mathrm{X}_{1}\right)$
b) Calidad del servicio proporcionado por los médicos $\left(\mathrm{X}_{2}\right)$
c) Calidad del servicio proporcionado por el personal de la farmacia $\left(\mathrm{X}_{3}\right)$
d) Calidad del servicio en la entrega de medicamentos $\left(\mathrm{X}_{4}\right)$
e) Calidad del servicio en la recibimiento y entrega de fichas $\left(X_{5}\right)$
f) Calidad del servicio con respecto a análisis clínicos $\left(\mathrm{X}_{6}\right)$
g) Calidad percibida de las instalaciones $\left(\mathrm{X}_{7}\right)$
h) Satisfacción de los derechohabientes (Y) 
Tabla 2

Análisis factorial confirmatorio de las dimensiones del modelo. Rotación oblicua (no ortogonal). Cargas factoriales (matriz patrón)

\begin{tabular}{|c|c|c|c|c|c|c|c|c|}
\hline \multirow[b]{2}{*}{ Atributos de calidad en el servicio } & \multicolumn{8}{|c|}{ Componentes } \\
\hline & 1 & 2 & 3 & 4 & 5 & 6 & 7 & 8 \\
\hline Nivel de experiencia de las enfermeras que lo atienden & .84 & & & & & & & \\
\hline Atención de la enfermera que lo atendió el día de hoy & .79 & & & & & & & \\
\hline Amabilidad de las enfermeras que lo atienden & .77 & & & & & & & \\
\hline Claridad de las explicaciones de las enfermeras & .75 & & & & & & & \\
\hline Nivel de conocimiento de la(o)s enfermera $(o) s$ & .75 & & & & & & & \\
\hline Nivel de conocimiento del médico que le atendió hoy & & .80 & & & & & & \\
\hline Nivel de experiencia del médico que lo(a) atendió hoy & & .75 & & & & & & \\
\hline Atención del médico que le atendió hoy & & .70 & & & & & & \\
\hline Amabilidad del médico que lo(a) atendió hoy & & .70 & & & & & & \\
\hline Claridad de las explicaciones del médico & & 69 & & & & & & \\
\hline $\begin{array}{l}\text { Entrega en la farmacia de dosis completa de medicamento } \\
\text { recetado }\end{array}$ & & & .83 & & & & & \\
\hline $\begin{array}{l}\text { Entrega en la farmacia de todo medicamento prescrito por el } \\
\text { médico }\end{array}$ & & & .82 & & & & & \\
\hline Buen estado del medicamento entregado & & & .78 & & & & & \\
\hline $\begin{array}{l}\text { Nivel de conocimiento del personal que realiza los análisis } \\
\text { clínicos }\end{array}$ & & & & -.87 & & & & \\
\hline Nivel de experiencia del personal que realiza los análisis clínicos & & & & -.79 & & & & \\
\hline $\begin{array}{l}\text { Claridad de las explicaciones del personal que realiza los análisis } \\
\text { clínicos }\end{array}$ & & & & -.70 & & & & \\
\hline Amabilidad del personal que realiza los análisis clínicos & & & & -.68 & & & & \\
\hline Atención del personal que le realizó análisis clínicos & & & & -.66 & & & & \\
\hline Claridad de las indicaciones del personal que dio las fichas/citas & & & & & .83 & & & \\
\hline Atención del personal que dio fichas/citas & & & & & .81 & & & \\
\hline Atención de las recepcionistas & & & & & .73 & & & \\
\hline Claridad de las indicaciones de las recepcionistas & & & & & .72 & & & \\
\hline Calidad del servicio recibido versus el ideal & & & & & & -.81 & & \\
\hline Satisfacción general con el servicio recibido el día de hoy & & & & & & -.80 & & \\
\hline Calidad del servicio recibido versus expectativas & & & & & & -.78 & & \\
\hline Calidad de la instalaciones de la Institución & & & & & & & .68 & \\
\hline Imagen de esta Institución & & & & & & & .64 & \\
\hline Limpieza de esta Institución & & & & & & & .63 & \\
\hline Comodidad de la Institución & & & & & & & .60 & \\
\hline Limpieza del equipo médico & & & & & & & .59 & \\
\hline Funcionalidad del equipo médico & & & & & & & .56 & \\
\hline Trato de los policías & & & & & & & & \\
\hline Amabilidad de la persona que lo(a) atiende en la farmacia & & & & & & & & .83 \\
\hline Atención del personal de farmacia que lo atendió hoy & & & & & & & & .80 \\
\hline $\begin{array}{l}\text { Nivel de conocimiento de la persona que lo(a) atendió en la } \\
\text { farmacia }\end{array}$ & & & & & & & & .71 \\
\hline
\end{tabular}

Solución de 35 variables a 8 componentes con eigenvalores superiores a 1 que explican el $70.3 \%$ de la variabilidad original. Método de extracción: Análisis de componentes principales. Método de rotación: Oblimin con normalización Kaiser. Rotación convergió en 16 iteraciones.

De estos ocho componentes, siete corresponden a las dimensiones de calidad en el servicio que fueron mencionados en la sección de planteamiento. Estas siete dimensiones de calidad en el servicio se utilizan aquí como variables latentes independientes en cada una de las siete hipótesis que fueron anteriormente planteadas. La dimensión número ocho (satisfacción) se correspondería a la variable latente dependiente en estas hipótesis. Los reactivos que se agrupan en estas ocho dimensiones (variables latentes) se pueden ver en la Tabla 3. 


\section{Mediciones}

Para confirmar la confiabilidad en las mediciones se realizaron análisis de fiabilidad utilizando el alfa de Cronbach. Para estos análisis se obtuvo el índice de fiabilidad de los reactivos (atributos) que conforman cada una de las dimensiones obtenidas en el análisis factorial de la Tabla 2 y que luego se utilizan como variables observadas en el análisis estructural. El alfa de Cronbach es un estadístico altamente confiable para determinar la consistencia interna de componentes de reactivos múltiples unidimensionales (Gliem y Gliem, 2003) tal y como se hace en el presente documento. En la Tabla 3 se puede observar que en los reactivos asociados a cada dimensión (variables latentes) se obtuvieron valores en el coeficiente alfa que pueden indicar altos niveles de consistencia interna en la medición según Darren y Mallery (2003, p. 231), y según Gliem y Gliem (2003).

Los reactivos utilizados para medir satisfacción son consistentes con aquellos utilizados en estudios previos de forma muy generalizada como puede verse en: Zeithaml et al. (1996), Oliver (1999), Baumann et al. (2007), y Ryu et al. (2012), así como en muchos otros. Aunque se han propuesto diferentes modelos para medir satisfacción, los reactivos utilizados en el presente estudio son consistentes con el enfoque propuesto por Westbrook (1980) donde la satisfacción se considera una respuesta afectiva global sobre la prestación del servicio. Esta respuesta afectiva sería resultante de la comparación entre el resultado obtenido de un servicio y una expectativa determinada con anterioridad (Halstead, Hartman y Schmidt, 1994).

\section{Resultados: análisis descriptivo y modelos de regresión}

En la Tabla 4 se muestran resultados descriptivos del estudio. En esta tabla se puede apreciar que se dieron diferencias significativas entre instituciones en la mayoría de cada uno de los atributos medidos. Los valores más altos fueron obtenidos entre dos de las tres instituciones utilizadas en este trabajo. Para confirmar diferencias entre las instituciones fueron utilizadas pruebas de diferencias para muestras independientes. Cada muestra independiente corresponde a los derechohabientes de cada una de las instituciones. Para este propósito, se utilizaron dos pruebas distintas, por un lado, una prueba paramétrica: prueba anova de un factor para diferencias de medias. Para confirmar las diferencias, y para evitar discusiones sobre el nivel de medición y la normalidad de los datos por provenir de escalas de actitud, se utilizó también una prueba no paramétrica: prueba Kruskal Wallis para diferencia de distribuciones. Como se puede apreciar en la Tabla 4 los resultados de ambas pruebas se confirman entre sí en todos los casos a excepción de una de las mediciones de satisfacción. 
Tabla 3

Reactivos (variables observables) por componentes (variables latentes) y fiabilidad de las mediciones

\begin{tabular}{|c|c|c|}
\hline $\begin{array}{l}\text { Dimensión } \\
\text { (variable latente) }\end{array}$ & $\begin{array}{l}\text { Indicador } \\
\text { (variable observada) }\end{array}$ & $\begin{array}{l}\text { Alfa } \\
(\alpha)\end{array}$ \\
\hline Enfermera(o)s & $\begin{array}{l}\text { ¿Cómo considera la atención de la enfermera que lo atendió el día de hoy? } \\
\text { ¿Cómo percibe el nivel de conocimiento de las enfermeras que lo(a) atendieron el } \\
\text { día de hoy? } \\
\text { ¿Cómo percibe el nivel de experiencia de las enfermeras que lo atienden? } \\
\text { ¿Cómo percibe la amabilidad de las enfermeras que lo atienden? } \\
\text { ¿Cómo considera la claridad de las explicaciones de las enfermeras que lo(a) } \\
\text { atienden? }\end{array}$ & .92 \\
\hline Médicos & $\begin{array}{l}\text { ¿Cómo considera la atención del médico que le atendió hoy? } \\
\text { ¿Cómo percibe el nivel de conocimiento del médico que le atendió hoy? } \\
\text { ¿Cómo percibe el nivel de experiencia del médico que lo(a) atendió hoy? } \\
\text { ¿Cómo percibe la amabilidad del médico que lo(a) atendió hoy? } \\
\text { ¿Cómo considera la claridad de las explicaciones del médico que lo(a) atendió hoy? }\end{array}$ & .93 \\
\hline $\begin{array}{l}\text { Personal de } \\
\text { la farmacia }\end{array}$ & $\begin{array}{l}\text { ¿Cómo considera la atención del personal de farmacia que lo atendió hoy? } \\
\text { ¿Cómo percibe el nivel de conocimiento de la persona que lo(a) atendió en la } \\
\text { farmacia? } \\
\text { ¿Cómo percibe la amabilidad de la persona que lo(a) atiende en la farmacia? }\end{array}$ & .92 \\
\hline $\begin{array}{l}\text { Entrega de } \\
\text { medicamentos }\end{array}$ & $\begin{array}{l}\text { Encontré todo el medicamento prescrito por el médico en la farmacia el día de hoy } \\
\text { Cuando me entregaron mi medicamento, se me entregó la dosis completa } \\
\text { El medicamento que me entregaron al terminar mi cita se encuentra en buen estado }\end{array}$ & .81 \\
\hline $\begin{array}{l}\text { Recibimiento y } \\
\text { entrega de fichas }\end{array}$ & $\begin{array}{l}\text { ¿Cómo considera la atención del personal que dio fichas/citas? } \\
\text { ¿Cómo considera la claridad de las indicaciones del personal que dio las fichas/ } \\
\text { citas? } \\
\text { ¿Cómo considera la atención de las recepcionistas? (personas que pasan, pesan y } \\
\text { miden) } \\
\text { ¿Cómo considera la claridad de las indicaciones de las recepcionistas? }\end{array}$ & .89 \\
\hline Análisis clínicos & $\begin{array}{l}\text { ¿Cómo considera la atención del personal que le realizó análisis clínicos hoy? } \\
\text { ¿Cómo percibe el nivel de conocimiento del personal que realiza los análisis } \\
\text { clínicos? } \\
\text { ¿Cómo percibe el nivel de experiencia del personal que realiza los análisis clínicos? } \\
\text { ¿Cómo percibe la amabilidad del personal que realiza los análisis clínicos? } \\
\text { ¿Cómo considera la claridad de las explicaciones del personal que realiza los } \\
\text { análisis clínicos? }\end{array}$ & .89 \\
\hline Instalaciones & $\begin{array}{l}\text { ¿Considera que las instalaciones (consultorios, sala de espera, baños, cafetería) son? } \\
\text { ¿Considera que la limpieza de esta Institución es? } \\
\text { ¿Considera que la imagen de esta Institución es? } \\
\text { ¿Considera que la comodidad de la Institución es? } \\
\text { ¿Cómo considera la funcionalidad del equipo médico? (camillas, básculas, } \\
\text { estetoscopio, sillas) } \\
\text { ¿Considera que la limpieza del equipo médico es? }\end{array}$ & .87 \\
\hline Satisfacción (Y) & $\begin{array}{l}\text { En general, me siento satisfecho con el servicio recibido el día de hoy } \\
\text { El servicio recibido el día de hoy cumplió con mis expectativas. } \\
\text { El servicio recibido el día de hoy fue el ideal. }\end{array}$ & .93 \\
\hline
\end{tabular}

Alfa $(\alpha)$ Coeficiente de fiabilidad interna alfa de Cronbach 
Tabla 4

Resultados por institución por reactivo. Medias, desviaciones estándar y pruebas de diferencias entre instituciones

\begin{tabular}{|c|c|c|c|c|c|c|c|c|}
\hline \multirow{3}{*}{ Reactivo (variable observada) } & \multicolumn{6}{|c|}{ Institución } & \multirow[b]{3}{*}{ auf } & \multirow[b]{3}{*}{$\mathrm{kw}$} \\
\hline & \multicolumn{2}{|c|}{ IMSS } & ISSSTE & \multicolumn{2}{|c|}{ SSA } & \multirow[b]{2}{*}{$\mathrm{s}$} & & \\
\hline & $\dot{\mathrm{x}}$ & $\mathrm{s}$ & $\dot{\mathrm{x}}$ & $\mathrm{s}$ & $\dot{\mathrm{x}}$ & & & \\
\hline Calidad las instalaciones de esta Institución & 4.13 & .88 & 4.02 & .73 & 4.08 & .99 & - & - \\
\hline Limpieza de esta Institución & 4.08 & .93 & 3.85 & .71 & 4.23 & .87 & $* * *$ & $* * *$ \\
\hline Imagen de esta Institución & 4.08 & .94 & 3.87 & .75 & 4.08 & .88 & $*$ & $* *$ \\
\hline Comodidad de las instalaciones & 4.00 & .86 & 3.77 & .80 & 3.90 & 1.10 & $*$ & $*$ \\
\hline Funcionalidad del equipo médico & 4.13 & .88 & 3.92 & .79 & 4.45 & .87 & $* * *$ & $* * *$ \\
\hline Limpieza del equipo médico & 4.27 & .77 & 3.97 & .72 & 4.55 & .73 & $* * *$ & $* * *$ \\
\hline Atención del médico que le atendió hoy & 4.59 & .86 & 4.25 & .78 & 4.80 & .82 & $* * *$ & $* * *$ \\
\hline $\begin{array}{l}\text { Nivel de conocimiento del médico } \\
\text { que le atendió hoy }\end{array}$ & 4.67 & .83 & 4.19 & .84 & 4.92 & .80 & $* * *$ & $* * *$ \\
\hline Nivel de experiencia del médico & 4.67 & .90 & 4.19 & .88 & 4.92 & .83 & $* * *$ & $* * *$ \\
\hline Amabilidad del médico & 4.60 & 1.01 & 4.18 & .92 & 4.78 & .83 & $* * *$ & $* * *$ \\
\hline Claridad de las explicaciones del médico & 4.57 & .96 & 4.15 & .90 & 4.72 & .84 & $* * *$ & $* * *$ \\
\hline Atención del personal que dio fichas/citas & 4.06 & 1.02 & 3.67 & .92 & 4.08 & .78 & $* * *$ & $* * *$ \\
\hline $\begin{array}{l}\text { Claridad de las indicaciones del personal } \\
\text { que dio las fichas/citas }\end{array}$ & 4.18 & .91 & 3.74 & .82 & 4.08 & .81 & $* * *$ & $* * *$ \\
\hline Atención de las recepcionistas & 4.23 & .88 & 3.78 & .86 & 4.05 & .73 & $* * *$ & $* * *$ \\
\hline Claridad de las indicaciones de las recepcionistas & 4.20 & .90 & 3.83 & .82 & 4.05 & 69 & $* * *$ & $* * *$ \\
\hline Atención de la enfermera que lo atendió & 4.33 & .81 & 4.18 & .81 & 4.49 & .77 & $* *$ & $* *$ \\
\hline $\begin{array}{l}\text { Nivel de conocimiento de las enfermeras } \\
\text { que lo(a) atendieron }\end{array}$ & 4.33 & .83 & 4.17 & .69 & 4.59 & .73 & $* * *$ & $* * *$ \\
\hline Nivel de experiencia de las enfermeras & 4.33 & .83 & 4.18 & 69 & 4.57 & .73 & $* * *$ & $* * *$ \\
\hline Amabilidad de las enfermeras & 4.28 & 1.07 & 4.17 & .79 & 4.47 & .77 & $* *$ & $* *$ \\
\hline Claridad de las explicaciones de las enfermeras & 4.32 & .93 & 4.10 & .77 & 4.46 & .71 & $* * *$ & $* * *$ \\
\hline Trato de los policías & 4.28 & .82 & 4.12 & .74 & 3.84 & 1.00 & $* * *$ & $* * *$ \\
\hline Atención del personal de farmacia & 4.09 & .96 & 3.84 & .82 & 4.33 & .62 & $* * *$ & $* * *$ \\
\hline Nivel de conocimiento de la persona en la farmacia & 4.09 & .95 & 3.89 & .79 & 4.26 & .72 & $* * *$ & $* * *$ \\
\hline Amabilidad de la persona en la farmacia & 3.94 & 1.10 & 3.84 & .87 & 4.39 & .61 & $* * *$ & $* * *$ \\
\hline $\begin{array}{l}\text { Atención del personal que le realizó análisis } \\
\text { clínicos }\end{array}$ & 4.40 & .86 & 3.96 & .76 & 4.36 & .65 & $* * *$ & $* * *$ \\
\hline $\begin{array}{l}\text { Nivel de conocimiento del personal que realiza } \\
\text { los análisis clínicos }\end{array}$ & 4.57 & .78 & 4.06 & .68 & 4.37 & .61 & $* * *$ & $* * *$ \\
\hline $\begin{array}{l}\text { Nivel de experiencia del personal que realiza } \\
\text { los análisis clínicos }\end{array}$ & 4.67 & .74 & 4.02 & .75 & 4.39 & .60 & $* * *$ & $* * *$ \\
\hline $\begin{array}{l}\text { Amabilidad del personal que realiza los análisis } \\
\text { clínicos }\end{array}$ & 4.38 & .75 & 4.05 & .76 & 4.35 & .59 & $* *$ & $* *$ \\
\hline $\begin{array}{l}\text { Claridad de las explicaciones del personal de } \\
\text { los análisis clínicos }\end{array}$ & 4.52 & .68 & 4.07 & .79 & 4.38 & .67 & $* * *$ & $* * *$ \\
\hline $\begin{array}{l}\text { Encontré medicamento prescrito por el médico } \\
\text { en la farmacia }\end{array}$ & 4.43 & 1.67 & 4.26 & 1.50 & 4.37 & 1.38 & - & - \\
\hline Medicamento, se me entregó dosis completa & 4.77 & 1.52 & 4.42 & 1.41 & 4.73 & 1.20 & $*$ & $* *$ \\
\hline $\begin{array}{l}\text { Medicamento que me entregaron se encuentra } \\
\text { en buen estado }\end{array}$ & 5.37 & 1.01 & 4.86 & 1.05 & 5.15 & .93 & $* * *$ & $* * *$ \\
\hline Satisfecho con el servicio recibido el día de hoy & 4.69 & 1.21 & 4.50 & 1.24 & 4.91 & .83 & $* *$ & $* *$ \\
\hline Servicio cumplió con mis expectativas. & 4.65 & 1.24 & 4.42 & 1.28 & 4.70 & .96 & $*$ & - \\
\hline Servicio recibido fue el ideal. & 4.40 & 1.29 & 4.11 & 1.29 & 4.62 & .99 & $* * *$ & $* * *$ \\
\hline
\end{tabular}

X்: media (medias en negritas indican el valor más alto por variable)

s: desviación estándar

Las medias corresponden a puntuaciones posibles entre 1 y 6 , donde 1 se refiere al valor más bajo y 6 al más alto. auf: Prueba de diferencias de medias Anova de un factor. Diferencias significativas *al .05, **al .01, ***al .001 kw: Prueba de Kruskal Wallis para varias muestras independientes. Diferencias significativas *al .05, **al .01, ***al .001 
Para contar con una primera aproximación a un análisis de causalidad se generaron tres modelos con análisis de regresión múltiple. Para estos modelos las variables dependientes utilizadas fueron las tres mediciones de satisfacción (variables observadas de satisfacción). Como variables independientes se utilizaron las siete dimensiones de calidad en el servicio sintetizadas mediante el análisis factorial con rotación oblicua que pueden verse en las Tablas 2 y 3 .

Tabla 5

Análisis de regresión lineal. Mediciones de satisfacción como variables dependientes. Coeficientes estandarizados.

\begin{tabular}{lcccc}
\hline & $\begin{array}{l}\text { Modelo 1 } \\
\text { Y, Me siento } \\
\text { satisfecho }\end{array}$ & $\begin{array}{c}\text { Modelo 2 } \\
\text { Y Cumplió } \\
\text { expectativas }\end{array}$ & $\begin{array}{c}\text { Modelo 3 } \\
\text { Y S Servicio } \\
\text { fue ideal }\end{array}$ & $\mathrm{Z}$ \\
\hline $\mathrm{R}$ & .62 & .63 & .50 & \\
$\mathrm{R}^{2}$ ajustada & .37 & .37 & .22 & \\
$\mathrm{~F}$ & $17.47^{* * *}$ & $17.65^{* * *}$ & $9.06^{* * * *}$ & \\
$\mathrm{X}_{1}$ Enfermeras & .06 & .12 & .07 & $1.56^{*}$ \\
$\mathrm{X}_{2}$ Médicos & $.22^{* *}$ & $.13^{*}$ & .12 & 1.19 \\
$\mathrm{X}_{3}$ Farmacia & $.17^{* *}$ & $.14^{*}$ & .11 & $1.48^{*}$ \\
$\mathrm{X}_{4}$ Medicamentos & $.34^{* * *}$ & $.31^{* * *}$ & $.20^{* *}$ & 0.96 \\
$\mathrm{X}_{5}$ Recepción/fichas & .04 & -.03 & $-.16^{*}$ & 1.01 \\
$\mathrm{X}_{6}$ Análisis clínicos & .08 & .09 & .12 & $1.71^{*}$ \\
$\mathrm{X}_{7}$ Instalaciones & $.25^{* * *}$ & $.27^{* * *}$ & .08 & 1.37 \\
\hline
\end{tabular}

*Significativo al .05, **Significativo al .01, ***Significativo al .001

Z: Prueba de normalidad Kolmogorov-Smirnov. H nula: La distribución es normal

Los resultados de estos análisis de regresión pueden verse en la Tabla 5. De las siete variables independientes cuatro resultaron significativas (médicos, farmacia, medicamentos e instalaciones) para dos de las mediciones de satisfacción (satisfacción general y cumplió expectativas). Para la tercera medición de satisfacción (ideal) sólo dos variables independientes resultaron significativas (medicamentos y recepción de fichas).

En la Tabla 5 también se muestran indicadores de normalidad de las variables independientes utilizadas en los modelos de regresión lineal. Estos indicadores muestran que no todas las variables independientes cumplen con el criterio de normalidad (aunque el alejamiento no es mucho). Esto podría generar suspicacias sobre la validez de estos análisis de regresión lineal, ya que la normalidad de las variables es uno de los supuestos básicos asociados al análisis de regresión y, aunque esto es argumentable, para algunos puede ser requisito indispensable. Para afrontar esta posible discusión, se determinó ofrecer adicionalmente análisis de regresión de tipo logística ordinal, que no asume linealidad ni el supuesto de normalidad. En la tabla 6 se pueden ver los resultados de tres modelos de regresión ordinal donde cada uno corresponde a las variables dependientes utilizadas en los modelos de regresión lineal de la Tabla 5. Los tres modelos de regresión ordinal en general muestran indicadores adecuados para aceptarlos. Así, se confirma que las variables independientes tienden a tener un efecto sobre las dependientes, y se obtiene que los datos se ajustan bien al modelo. Sólo con respecto a la prueba de líneas paralelas, el Modelo 6 no aprobó este criterio. 
Tabla 6

Análisis de regresión logística ordinal. Mediciones de satisfacción como variables dependientes. Indicadores del modelo. Pesudo-coeficientes de determinación $\mathrm{R}^{2}$. Parámetros estimadores de las variables independientes.

\begin{tabular}{lccc}
\hline & Modelo 4 & Modelo 5 & Modelo 6 \\
& Y $_{1}$ Me siento satisfecho & Y $_{2}$ Cumplió expectativas & Y $_{3}$ Servicio fue ideal \\
\hline Ajuste del modelo & $101.2^{* * * *}$ & $99.1 * * *$ & $52.5^{* * * *}$ \\
Bondad de ajuste Pearson & 785.8 & 996.4 & 867.8 \\
Bondad de ajuste Deviance & 455.9 & 468.7 & 524.8 \\
Parallel & 31.06 & 16.5 & $48.9 * *$ \\
$\mathrm{R}^{2}$ Cox y Snell & .40 & .39 & .23 \\
$\mathrm{R}^{2}$ Nagelkerke & .42 & .41 & .24 \\
$\mathrm{R}^{2}$ McFadden & .18 & .18 & .09 \\
$\mathrm{X}_{1}$ Enfermeras & .17 & $.38^{*}$ & .20 \\
$\mathrm{X}_{2}$ Médicos & $.59^{* * *}$ & .28 & .24 \\
$\mathrm{X}_{3}$ Farmacia & $.46^{* * *}$ & $.41^{* *}$ & $.27 *$ \\
$\mathrm{X}_{4}$ Medicamentos & $.87^{* * *}$ & $.81^{* * *}$ & $.45^{* *}$ \\
$\mathrm{X}_{5}$ Recepción/fichas & .17 & -.00 & $-.34^{*}$ \\
$\mathrm{X}_{6}$ Análisis clínicos & .15 & .21 & .22 \\
$\mathrm{X}_{7}$ Instalaciones & $.66^{* * *}$ & $.66^{* * *}$ & .12 \\
\hline
\end{tabular}

Ajuste del modelo: Prueba ji2 para determinar que existe efecto de las variables independientes (debe ser significativa) Bondad de ajuste Pearson: prueba para determinar si los datos se ajustan al modelo (debe ser no significativa)

Bondad de ajuste Deviance: prueba para determinar si los datos se ajustan al modelo (debe ser no significativa)

Parallel: prueba de líneas paralelas para determinar igualdad de explicación sobre los valores (debe ser no significativa)

R2 C\&S: coeficiente R2 de Cox y Snell para determinar grado de explicación sobre la dependiente $(0$ a $1 ; .5 \approx 50 \%)$

R2 N: coeficiente R2 de Nagelkerke para determinar grado de explicación sobre la dependiente $(0$ a $1 ; .5 \approx 50 \%)$

R2 Mc: coeficiente R2 de McFadden para determinar grado de explicación sobre la dependiente $(0$ a $1 ; .5 \approx 50 \%)$

Estimadores de las variables independientes deben ser significativos para concluir efecto sobre la dependiente

*significancia a $0.05 / * *$ significancia a $0.01 / * * *$ Significativo al .001

En términos generales los resultados de los modelos de regresión ordinal confirman a los de los modelos de regresión lineal. Los coeficientes de determinación tienden a ser similares en ambos tipos de modelos. También, las variables independientes que tienen efecto significativo sobre las dependientes tienden a ser las mismas en ambos tipos de regresiones. Esto puede sugerir que cuando no se tiene un alejamiento severo del supuesto de normalidad en una muestra grande, aunque el valor de la prueba $\mathrm{Z}$ sea significativo, la regresión lineal puede funcionar.

\section{Resultados del análisis estructural}

Se hicieron dos modelos estructurales (modelaje de ecuaciones estructurales) utilizando el software Amos. Inicialmente se programó el modelo completo contemplando las siete hipótesis planteadas donde se relaciona las siete variables latentes de calidad del servicio con la variable latente de satisfacción (ver Figura 1). Se utilizaron los datos obtenidos de la aplicación de cuestionarios como variables observadas. Estas variables observadas se relacionaron con cada una de sus respectivas ocho variables latentes de acuerdo al análisis factorial que se muestra en la Tabla 1. Posteriormente, se identificaron qué variables latentes independientes tienen un impacto significativo en la satisfacción, y cuáles no. Con esto se generó el segundo modelo estructural que se le llamó modelo final. 
Se considera importante mencionar aquí que al realizar la prueba de normalidad de todas las variables observadas con las que se alimentó a los modelos estructurales, ninguna cumple con el criterio de normalidad (con la prueba de Kolmogorov-Smirnov). Se supone que el criterio de normalidad es un supuesto requerido en el análisis estructural. Sin embargo, existen evidencias que sugieren que cuando se tienen muestras muy grandes ( $n=100$ o mayor), como en este caso, la no normalidad de las variables observadas tiende a no afectar la exactitud del modelo estructural (Jannoo, Auchoybur y Lazim, 2014).

En la Tabla 7 se puede ver que a excepción del criterio de chi-cuadrada, los modelos estructurales cumplen satisfactoriamente con todos los indicadores de ajuste requeridos para considerarlos como modelos aceptables. Los coeficientes de ajuste a la realidad (NFI, IFI, TLI y CFI) arriba de 0.90 se consideran aceptables, y por encima de 0.95 como buenos (Bentler y Bonett, 1980). Valores de 0.08 o inferiores en el RMSEA se consideran aproximaciones de error razonables (aceptables) y valores de 0.05 o menores indicarían un buen ajuste con respecto a los grados de libertad, así se sugiere no aceptar valores mayores a 0.1 (Browne y Cudeck, 1993; MacCallum et al., 1996). En el indicador CMIN/DF (chi-cuadrada dividida entre los grados de libertad) valores menores a 5.0 pueden considerarse aceptables (Wheaton et al., 1977; Marsh y Hocevar, 1985). El RMSEA y el CMI/DF son medias alternativas de ajuste para aceptar el modelo cuando la chi-cuadrada es demasiado grande como en este caso (Browne y Cudeck, 1993; MacCallum et al., 1996; Kenny, 2011).

Tabla 7

Modelos estructurales. Indicadores de ajuste.

\begin{tabular}{lcc}
\hline & Modelo inicial & Modelo final \\
\hline $\mathrm{Chi}^{2}$ & $1340.5^{* * *}$ & $680.6^{* * *}$ \\
$\mathrm{CMIN} / \mathrm{DF}$ & 2.69 & 3.42 \\
$\mathrm{NFI}$ & .90 & .91 \\
IFI & .94 & .94 \\
TLI & .92 & .92 \\
CFI & .94 & .94 \\
RMSEA & .053 & .064 \\
\hline
\end{tabular}

En la Tabla 8 se pueden ver los resultados de los pesos de regresión entre las variables latentes en los modelos estructurales. En el primer modelo se introdujeron todas las variables latentes independientes (dimensiones de calidad del servicio) para determinar si existe un efecto sobre la variable latente de satisfacción del derechohabiente. Aquí, sólo tres variables latentes independientes mostraron pesos de regresión estadísticamente significativos hacia la satisfacción: servicio del médico, entrega de medicamentos e instalaciones. Entrega de medicamentos con el valor del peso de regresión más alto.

Para el modelo estructural final se fueron eliminando variables latentes independientes no significativas de forma paulatina comenzando por la del peso de regresión más bajo. Al final una variable latente independiente que no había sido significativa en el modelo inicial resultó significativa en el modelo final. De tal forma se obtiene un modelo final depurado (más parsimonioso) sin pesos de regresión no significativos con cuatro variables latentes independientes significativas: servicio del médico, entrega de medicamentos, análisis clínicos y calidad de las instalaciones. El atributo entrega de medicamentos otra vez con el peso de regresión más alto. En la Tabla 9 se puede ver un comparativo entre los coeficientes de determinación y los coeficientes de regresión (parámetros o estimadores) de todos los análisis realizados. 
Tabla 8

Análisis estructurales. Pesos de regresión estandarizados y coeficientes de determinación $\mathrm{R}^{2}$ para la variable dependiente (satisfacción)

\begin{tabular}{lll}
\hline Parámetros & Modelo inicial & Modelo final \\
\hline Enfermeras ---> Satisfacción & -.003 & -- \\
Médicos ---> Satisfacción & $.283^{* * *}$ & $.279 * *$ \\
Personal farmacia ---> Satisfacción & .013 & -- \\
Medicamentos ---> Satisfacción & $.383^{* * *}$ & $.379 * *$ \\
Recepción/fichas ---> Satisfacción & .034 & -- \\
Análisis clínicos ---> Satisfacción & .133 & $.157 *$ \\
Instalaciones ---> Satisfacción & $.130^{*}$ & $.145^{* *}$ \\
\hline $\mathrm{R}^{2}$ para Satisfacción & .50 & .49
\end{tabular}

*Significativo al .05, **Significativo al .01, ***Significativo al .001

$\mathrm{R}^{2}=$ cuadrado de la correlación múltiple para la variable dependiente

Tabla 9

Coeficientes de determinación y coeficientes de regresión

\begin{tabular}{|c|c|c|c|c|c|}
\hline & & $\begin{array}{l}\text { Me siento } \\
\text { satisfecho }\end{array}$ & $\begin{array}{l}\text { Cumplió } \\
\text { expectativas }\end{array}$ & $\begin{array}{l}\text { Servicio fue } \\
\text { ideal }\end{array}$ & $\begin{array}{l}\text { Satisfacción } \\
\text { (latente) }\end{array}$ \\
\hline $\begin{array}{l}\text { Regresión } \\
\text { lineal }\end{array}$ & $\begin{array}{l}\mathrm{R}^{2} \\
\mathrm{X}_{1} \text { Enfermeras } \\
\mathrm{X}_{2} \text { Médicos } \\
\mathrm{X}_{3} \text { Farmacia } \\
\mathrm{X}_{4} \text { Medicamentos } \\
\mathrm{X}_{5} \text { Recepción/fichas } \\
\mathrm{X}_{6} \text { Análisis clínicos } \\
\mathrm{X}_{7} \text { Instalaciones }\end{array}$ & $\begin{array}{l}.37 \\
.06 \\
.22 * * \\
.17 * * \\
.34 * * * \\
.04 \\
.08 \\
.25 * * *\end{array}$ & $\begin{array}{l}.37 \\
.12 \\
.13 * \\
.14 * \\
.31 * * * \\
-.03 \\
.09 \\
.27 * * *\end{array}$ & $\begin{array}{l}.22 \\
.07 \\
.12 \\
.11 \\
.20^{* *} \\
-.16^{*} \\
.12 \\
.08\end{array}$ & \\
\hline $\begin{array}{l}\text { Regresión } \\
\text { ordinal }\end{array}$ & $\begin{array}{l}\mathrm{R}^{2} \text { Cox y Snell } \\
\mathrm{R}^{2} \text { Nagelkerke } \\
\mathrm{R}^{2} \text { McFadden } \\
\mathrm{X}_{1} \text { Enfermeras } \\
\mathrm{X}_{2} \text { Médicos } \\
\mathrm{X}_{3} \text { Farmacia } \\
\mathrm{X}_{4} \text { Medicamentos } \\
\mathrm{X}_{5} \text { Recepción/fichas } \\
\mathrm{X}_{6} \text { Análisis clínicos } \\
\mathrm{X}_{7} \text { Instalaciones }\end{array}$ & $\begin{array}{l}.40 \\
.42 \\
.18 \\
.17 \\
.59 * * * \\
.46^{* * *} \\
.87 * * * \\
.17 \\
.15 \\
.66^{* * * *}\end{array}$ & $\begin{array}{l}.39 \\
.41 \\
.18 \\
.38 * \\
.28 \\
.41 * * \\
.81 * * * \\
-.00 \\
.21 \\
.66 * * *\end{array}$ & $\begin{array}{l}.23 \\
.24 \\
.09 \\
.20 \\
.24 \\
.27^{*} \\
.45^{* *} \\
-.34^{*} \\
.22 \\
.12\end{array}$ & \\
\hline $\begin{array}{l}\text { Análisis } \\
\text { estructural } \\
\text { (modelo } \\
\text { final) }\end{array}$ & $\begin{array}{l}\mathrm{R}^{2} \\
\mathrm{X}_{1} \text { Enfermeras } \\
\mathrm{X}_{2} \text { Médicos } \\
\mathrm{X}_{3} \text { Farmacia } \\
\mathrm{X}_{4} \text { Medicamentos } \\
\mathrm{X}_{5} \text { Recepción/fichas } \\
\mathrm{X}_{6} \text { Análisis clínicos } \\
\mathrm{X}_{7} \text { Instalaciones }\end{array}$ & & & & $\begin{array}{l}.50 \\
-- \\
.28 * * \\
-- \\
.38^{* *} \\
-- \\
.16^{*} \\
.15^{* *}\end{array}$ \\
\hline
\end{tabular}

*Significativo al .05, **Significativo al .01, ***Significativo al .001

Nota: los coeficientes de determinación $\mathrm{R}^{2}$ no tienen asociado una prueba de significancia 


\section{Discusión y conclusiones}

A diferencia de estudios anteriores sobre medición de calidad del servicio en centros de salud. En el presente estudio se partió de una indagación exploratoria donde se realizó una detección muy detallada de atributos. De tal forma aquí se trabajó con 32 atributos muy específicos de calidad del servicio en el sector de clínicas de primer nivel de instituciones del Estado (ver Tabla 2). Enfoques anteriores (Babakus y Mangold, 1992; Murti et al., 2013) han retomado el instrumento SERVQUAL (Parasuraman et al., 1985) adaptando sus reactivos al servicio de centros de salud. Esta batería mide dimensiones generalizables a cualquier tipo de servicio como: tangibles, confiabilidad, respuesta, aseguramiento y empatía. Si bien el uso del SERVQUAL permite una mayor generalización de las conclusiones por este carácter estándar, aquí se considera que también ofrece menor explicación del fenómeno en particular bajo estudio. El haber detectado atributos muy específicos con base en el análisis del proceso de servicio que sigue un derechohabiente permite ofrecer una explicación más detallada de los generadores de satisfacción para los usuarios.

Estudios anteriores sobre medición de calidad del servicio en centros de salud, a diferencia del presente estudio, se han abocado a centros privados. En la revisión de literatura no se encontraron estudios de calidad del servicio realizados en clínicas públicas, al menos no en literatura de investigación académica. De esta forma, el presente estudio podría constituir el ofrecimiento de una primera visión, a este nivel de publicación, de lo que es la calidad del servicio en este tipo de instituciones.

De las siete hipótesis planteadas, en el presente estudio, no se encontraron evidencias para soportar las hipótesis $\mathrm{H}_{1}, \mathrm{H}_{3}$ y $\mathrm{H}_{5}$, lo que implica que factores como servicio de las enfermeras, servicio del personal de farmacia y servicio de recepción y entrega de fichas (lugares) pueden tener un peso secundario en la determinación de la percepción general de satisfacción del derechohabiente. Sin embargo, debe considerarse que, según estudios previos, si bien sólo algunos atributos son altamente importantes para generar alta satisfacción, un mal desempeño de los atributos de importancia secundaria pueden generar una disminución aun mayor de la satisfacción cuando el desempeño de los atributos principales está fallando (Myers y Alpert, 1968; Anderson y Sullivan, 2000). En otras palabras, estos atributos secundarios ayudan a mitigar la sensación de insatisfacción cuando fallan los atributos principales. Por lo anterior, estos atributos de preponderancia secundaria no debieran ser descartados de los análisis ni de la toma de decisiones en centros públicos de salud.

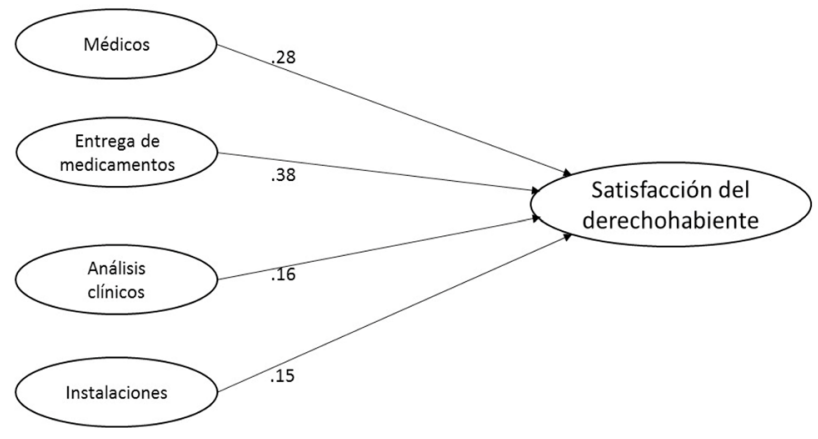

Figura 2. Modelo final y pesos de regresión estandarizados 
Con base en los resultados obtenidos, se puede argumentar que se tienen evidencias para soportar las hipótesis: $\mathrm{H}_{2}, \mathrm{H}_{4}, \mathrm{H}_{6}$ y $\mathrm{H}_{7}$. Por lo que se puede decir que probablemente los factores: servicio proporcionado por los médicos, servicio en la entrega de medicamentos, servicio del área de análisis clínicos, y la calidad de las instalaciones, son los de importancia principal para generar una sensación de alta satisfacción por parte de los derechohabientes (ver Tabla 9). De estos, aparentemente el servicio en la entrega de los medicamentos juega un papel más preponderante que los otros, inclusive por encima del servicio proporcionado por el médico. Entrega de medicamentos fue un factor que obtuvo los pesos estadísticos más altos tanto en los modelos de regresión como en los modelos estructurales (ver Tabla 9). Al principio de este estudio, se pensó que el servicio de los médicos debía ser el de mayor peso. Sin embargo, aparentemente tiende a ser la confianza en el medicamento el elemento último que puede estar permitiendo al usuario la sensación de que se va a curar y por lo tanto en donde concentra su percepción de valor hacia la institución. No obstante, el servicio del médico, aunque no fue el de mayor peso, sí el del segundo. Murti et al. (2013) anteriormente había propuesto conceptualmente que la calidad en la entrega del medicamento debía ser un atributo de relevancia secundaria en servicios de salud. Sin embargo, no ofreció evidencia empírica de ello. Los resultados del presente estudio estarían dando una confirmación empírica de lo contrario.

En los resultados de este estudio se dieron dos contradicciones entre los dos tipos de análisis que se realizaron (regresión versus estructural). Una de las contradicciones consiste en qué la variable "atención del personal de farmacia" salió significativa en dos de los tres modelos de regresión, pero no en ninguno de los dos modelos estructurales. La otra incongruencia consiste en que la variable "servicio del personal de análisis clínicos" no resultó significativa en ninguno de los modelos de regresión, pero sí en ambos modelos estructurales. Considerando que el análisis de ecuaciones estructurales en general es un método estadístico multivariado mucho más robusto que el análisis de regresión, aquí se determinó aceptar primordialmente los resultados del primero. De cualquier forma, siempre es interesante ver como en algunas ocasiones los resultados de dos métodos estadísticos distintos no necesariamente coinciden ayudando a incrementar las posibles interpretaciones.

Una limitación clara del presente estudio radica en que se llevó a cabo con derechohabientes que se atienden en clínicas de primer nivel en la Ciudad de México. En trabajos posteriores se podrían realizar estudios de calidad del servicio en clínicas de instituciones de salud de Estado en otras regiones del país para poder examinar diferencias geográficas. También, se podría realizar estudios de este tipo en centros especializados de segundo y tercer nivel. Como otra limitante se puede mencionar que este estudio no incluyó a otras instituciones públicas de salud como las que dependen de la SEDENNA, de PEMEX, del Seguro Popular, además de instituciones dependientes de gobiernos estatales como el ISSEMYM.

Con este estudio se ofrece un primer planteamiento sobre la relación directa entre dimensiones de calidad del servicio y la satisfacción de los derechohabientes del sector público de salud en México. Partiendo de este primer esbozo a futuro se podrían abordar la búsqueda de variables moderadoras de esta relación. El encuentro de variables moderadores permitirá ir generando un entendimiento más amplio del fenómeno de la satisfacción de los derechohabientes. Así, estas variables moderadoras nuevas permitirían incrementar los niveles de explicación de la variable dependiente (satisfacción del derechohabiente). Posibles hipótesis de variables moderadoras de esta relación pueden provenir de variables relacionadas con el constructo involucramiento. 
En el involucramiento se miden variables relacionadas a la percepción de la persona en cuanto a elementos como la importancia del servicio, su valor simbólico y el riesgo percibido en el mismo (Laurent y Kapferer, 1985; Kapferer y Laurent, 1993). Anteriormente se han publicado estudios (en otros sectores distintos al de instituciones de salud) sobre satisfacción y su relación con otros constructos que muestran que el involucramiento puede tener un efecto moderador (Sivakumar y Raj, 1997). Así, el riesgo que perciba un cliente en ser tratado en una clínica del Estado puede afectar su sensación de satisfacción muy a pesar de que el servicio recibido cumpla adecuadamente. Adicionalmente, se ha postulado anteriormente que el riesgo percibido puede tener en efecto mediador en la relación entre calidad percibida por el servicio o producto y la percepción de valor del usuario (Snoj et al., 2004). De esta forma, en estudios futuros, también se puede abordar la relación entre satisfacción del derechohabiente y su percepción de valor hacia este tipo de instituciones versus la posibilidad de tratarse en clínicas privadas.

Las variables utilizadas en este estudio están muy enfocadas a la atención, disposición y conocimientos del personal de las diferentes áreas, ya que estos fueron los aspectos que se obtuvieron en el primer acercamiento con los usuarios en un estudio cualitativo; sin embargo podría valer la pena incluir en investigaciones futuras atributos relacionados a los procedimientos en sí, por ejemplo el tiempo de respuesta ante alguna solicitud o el tiempo de espera para ser atendidos; así como la confianza en los resultados de los análisis de laboratorio, por mencionar algunos.

\section{Agradecimientos}

Se desea agradecer a IMS Health México por su importante participación en este proyecto y por consentir permitirnos utilizar los datos que sirvieron de base a este artículo. De forma particular se desea agradecer a Rosa Álvarez, Joselyn Chanes y Ricardo Moyano, colaboradores de IMS Health México Primary Intelligence, por su significativa contribución en el diseño del instrumento de medición que fue utilizado en este trabajo. Adicionalmente se extienden sendos reconocimientos a Mariel Corona, Graciela González, Mónica Rodríguez, Karla Rangel, José Arévalo, Itzel Morales, David Vega, David Charola, Melisa Trejo, Rosa Villanueva, Mario Alvarez, Sibila Guzmán, Diana Cabrera, Noemí García, Andrés Reyes, Karla Lara y María Aguilar. Ellos son alumnos del Tecnológico de Monterrey que aportaron de forma muy comprometida sus servicios en las labores para hacer de este proyecto de investigación una realidad.

\section{Referencias}

Akbaba, A. (2006). Measuring Service Quality in the Hotel Industry: A Study in a Business Hotel in Turkey. International Journal of Hospitality Management, 25(2): 170-192.

Amin, M. y Siti, Z. N. (2013). Hospital service quality and its effects on patient satisfaction and behavioural intention. Clinical Governance, 18(3): 238-254. DOI : CGIJ-05-2012-0016

Andaleeb, S. S. (2001). Service quality perceptions and patient satisfaction: a study of hospitals in a developing country. Social science \& medicine, 52(9): 1359-1370. http://dx.doi.org/10.1016/S0277-9536(00)00235-5

Anderson, E.W. y Sullivan M. (2000). The impact of critical incidents on customer satisfaction in business-to-business relationships. Journal of Business-to-Business Marketing, 8(1): 25-54.

Asubonteng, P., Mc Cleary, K. J. and Swan, J. E. (1996). SERVQUAL Revisited: A Critical Review of Service Quality. The Journal of Services Marketing, 10(6): 62-81. http://dx.doi.org/10.1108/08876049610148602 
Atta, O. E. G. (2012). The efficacy of applying the quality measure of health service on the external patient satisfaction. Management Review: An International Journal, 7(2): 81-110.

Babakus, E. y Boller, G.W. (1992). An empirical assessment of the SERVQUAL scale. Journal of Business Research, 24(3): 253-268. http://dx.doi.org/10.1016/0148-2963(92)90022-4

Babakus, E. y Mangold, W. G. (1992). Adapting the SERVQUAL scale to hospital services: an empirical investigation. Health Services Research, 26(6): 767-786.

Bansal, H. S. y Taylor, S. (2015). Investigating the relationship between service quality, satisfaction and switching intentions. Proceedings of the 1997 Academy of Marketing Science (AMS) Annual Conference: 304-313. Springer International Publishing. http://dx.doi.org/10.1007/978-3-319-13141-2_107

Baumann, C., Burton, S., Elliott, G. y Kehr, H.M. (2007). Prediction of attitude and behavioural intentions in retail banking. International Journal of Bank Marketing, 25(2): 102-116. http://dx.doi.org/10.1108/02652320710728438

Bentler, P.M. y Bonett, D.G. (1980). Significance tests and goodness of fit in the analysis of covariance structures. Psychological Bulletin, 88(1): 588-606. http://dx.doi.org/10.1037/0033-2909.88.3.588

Browne, M.W. y Cudeck, R. (1993). Alternative ways of assessing model fit. In Bollen, K.A. \& Long, J.S. [Eds.] Testing structural equation models. Newbury Park, CA: Sage: 136-162.

Bruwer, J. (2014). Service quality perception and satisfaction: Buying behaviour prediction in an Australian Festivalscape. International Journal of Tourism Research, 16(1): 76-86. http://dx.doi.org/10.1002/jtr.1901

Caro, L. y García, J.A. (2007). Cognitive-affective model of consumer satisfaction. An exploratory study within the framework of a sporting event. Journal of Business Research, 60(2): 108-114.

Chi Cui, C., Lewis, B. R., y Park, W. (2003). Service quality measurement in the banking sector in South Korea. The International Journal of Bank Marketing, 21(4/5), 191-201. http://dx.doi.org/10.1108/02652320310479187

Cronin, J.J. y Taylor, S.A. (1994). SERVPERF versus SERVQUAL; reconciling performance-based. Journal of Marketing, 58(1), 125-131.

Dabholkar, P. A., Thorpe, D. I. y Rentz, J. O. (1996). A measure of service quality for retail stores: scale development y validation. Journal of the Academy of Marketing Science, 24(1): 3-16. http://dx.doi.org/10.1007/BF02893933

Dagger, T. S., Sweeney, J. C., Johnson, L. W., (2007). A hierarchical model of health service quality: Scale development and investigation of an integrated model. Journal of Service Research, 10(2): 123-142. http://dx.doi. org/10.1177/1094670507309594

De Oña, R., Machado, J. L. y De Oña, J. (2015). Perceived Service Quality, Customer Satisfaction, and Behavioral Intentions: A Structural Equation Model for the Metro of Seville, Spain. In Transportation Research Board 94th Annual Meeting, No. 15-0897.

Donnelly, M., Wisniewski, M., Dalrymple, J. F. y Curry, A. C. (1995). Measuring service quality in local government: the SERVQUAL approach. International Journal of Public Sector Management, 8(7): 15-20. http://dx.doi. org/10.1108/09513559510103157

George, D. y Mallery, P. (2003). SPSS for Windows step by step: A simple guide and reference. 11.0 update. 4th ed., Boston: Allyn \& Bacon.

Gliem, J. A. y Gliem, R. R. (2003). Calculating, interpreting, and reporting Cronbach's alpha reliability coefficient for Likert-type scales. Midwest Research-to-Practice Conference in Adult, Continuing, and Community Education, Columbus, OH: 82-88.

Gómez, O., Sesma, S., Becerril, V. M., Knaul, F. M., Arreola, H., y Frenk, J. (2011). Sistema de salud de México. Salud pública de México, 53(1): 220-232.

Gounaris, S. (2005). An alternative measure for assessing perceived quality of software house services. The Service Industries Journal, 25(6): 803-823. DOI: 10.1080/02642060500103373

Grönroos, C. (1984). A service quality model and its marketing implications. European Journal of Marketing, 18(4): 36-44. DOI: 10.1108/EUM0000000004784 
Halstead, D., Hartman, D. y Schmidt, S. L. (1994). Multisource effects on the satisfaction formation process. Academy of Marketing Science Journal, 22(2): 114-129. http://dx.doi.org/10.1177/0092070394222002

Haque, A., Sarwar, A. A. M., Yasmin, F., Anwar, A., y Nuruzzaman. (2012). The impact of customer perceived service quality on customer satisfaction for private health centre in malaysia: A structural equation modeling approach. Information Management and Business Review, 4(5): 257-267.

Hill, F. M. y McCrory, M. L. (1997). An attempt to measure service quality at a belfast maternity hospital: Some methodological issues and some results. Total Quality Management, 8(5): 229-242. http://dx.doi. org/10.1080/0954412979488

Jabnoun, N. y Khalifa, A. (2005). A customized measure of service quality in the UAE. Managing Service Quality, 15(4): 374-388. DOI: 10.1108/09604520510606844

Jannoo, Z., Yap, B. W., Auchoybur, N., y Lazim, M. A. (2014). The Effect of Nonnormality on CB-SEM and PLSSEM Path Estimates. International Journal of Mathematical, Computational, Natural and Physical Engineering, 8(2): 285-291.

John, J. (1992). Patient satisfaction: The impact of past experience. Journal of Health Care Marketing, 12(3): 56-64

Kapferer, J. N. y Laurent, G. (1993). Further evidence on the consumer involvement profile: five antecedents of involvement. Psychology \& Marketing, 10(4): 347-355. http://dx.doi.org/10.1002/mar.4220100408

Kesuma, I. A. W., Hadiwidjojo, D., Wiagustini, N. L. P. y Rohman, F. (2013). Service quality influence on patient loyalty: Customer relationship management as mediation variable (study on private hospital industry in denpasar). International Journal of Business and Commerce, 2(12): 1-14.

Kenny, D. A. (2011). Measuring model fit. Obtenido el 19 de febrero de 2015. Disponible en: http://davidakenny.net/ $\mathrm{cm} /$ fit.htm

Kerlinger, F. N. (1979). Behavioral research: A conceptual approach. New York : Holt, Rinehart, and Winston.

Knutson, B., Stevens, P., Wullaert, C. y Yokoyama, F. (1991). Lodgserv: a service quality index for the lodging industry. Hospitality Research Journal, 14(3): 277-84.

Kumaraswamy, S. (2012). Service quality in health care centers: An empirical study. International Journal of Business and Social Science, special issue, 3(16): 141-150.

Laurent, G. y Kapferer, J. N. (1985). Measuring consumer involvement profiles. Journal of Marketing, 22(1): 41-53. http://dx.doi.org/10.2307/3151549

Lis, C. G., Rodeghier, M. y Gupta, D. (2011). The relationship between perceived service quality and patient willingness to recommend at a national oncology hospital network. BMC Health Services Research, 11(1): 1-8. http:// dx.doi.org/10.1186/1472-6963-11-46

MacCallum, R. C., Browne, M. W. y Sugawara, H. M. (1996). Power analysis and determination of sample size for covariance structure modeling. Psychological methods, 1(2): 130-149. http://dx.doi.org/10.1037/1082-989X.1.2.130

Marsh, H.W. y Hocevar, D. (1985). Application of confirmatory factor analysis to the study of self-concept: First- and higher-order factor models and their invariance across groups. Psychological Bulletin, 97(1): 562-582. http:// dx.doi.org/10.1037/0033-2909.97.3.562

Murti, A., Deshpande, A. y Srivastava, N. (2013). Service Quality, Customer (Patient) Satisfaction and Behavioural Intention in Health Care Services: Exploring the Indian Perspective. Journal of Health Management, 15(1): $29-44$. http://dx.doi.org/10.1177/0972063413486035

Myers, J.H. y Alpert, M.I. (1968). Determinant Attributes: Meaning and Measurement. Journal of Marketing, 32(4): 13-20.

Olin, S. S., Williams, N., Pollock, M., Armusewicz, K., Kutash, K., Glisson, C. y Hoagwood, K. E. (2014). Quality indicators for family support services and their relationship to organizational social context. Administration and policy in mental health and mental health services research, 41(1): 43-54. http://dx.doi.org/10.1007/s10488-0130499-z 
Oliver, R. (1980). A Cognitive Model of the Antecedents and Consequences of Satisfaction Decisions. Journal of Marketing Research, 17 (4): 460-469. http://dx.doi.org/10.2307/3150499

Oliver, R. (1999). Whence customer loyalty?. Journal of Marketing, 63(special issue): 33-44.

Orel, F. D. y Kara, A. (2014). Supermarket self-checkout service quality, customer satisfaction, and loyalty: Empirical evidence from an emerging market. Journal of Retailing and Consumer Services, 21(2): 118-129.

Parasuraman, A., Zeithaml, V. A. y Berry, L. L. (1985). A conceptual model of service quality and its implications for future research. Journal of Marketing, 49(1): 41-50. http://dx.doi.org/10.2307/1251430

Parasuraman, A., Zeithaml, V. A. y Berry, L. L. (1988). SERVQUAL: a multiple item scale for measuring consumer perceptions of service quality. Journal of Retailing, 64(1): 12-40.

Ryu, K., y Han, H. (2010). Influence of the quality of food, service, and physical environment on customer satisfaction and behavioral intention in quick-casual restaurants: Moderating role of perceived price. Journal of Hospitality \& Tourism Research, 34(2/3): 310-329. http://dx.doi.org/10.1177/1096348009350624

Ryu, K., Lee, H. y Kim, W. (2012). The influence of the quality of the physical environment, food, and service on restaurant image, customer perceived value, customer satisfaction, and behavioral intentions. International Journal of Contemporary Hospitality Management, 24(2): 200-223. http://dx.doi.org/10.1108/09596111211206141

Scott, D. y Shieff, D. (1993). Service quality components and group criteria in local government. International Journal of Service Industry Management, 4(4): 42-53. http://dx.doi.org/10.1108/09564239310044280

Sivakumar, K. y Raj, S. P. (1997). Quality tier competition: How price change influences brand choice and category choice. The Journal of Marketing, 61(3): 71-84. http://dx.doi.org/10.2307/1251790

Snoj, B., Korda, A. P. y Mumel, D. (2004). The relationships among perceived quality, perceived risk and perceived product value. Journal of Product \& Brand Management, 13(3): 156-167. http://dx.doi.org/10.1108/10610420410538050

Spreng, R. y R. Mackoy (1996). An empirical examination of a model of perceived service quality and satisfaction. Journal of Retailing, 72(2): 201-214. http://dx.doi.org/10.1016/S0022-4359(96)90014-7

Stevens, P., Knutson, B. y Patton, M. (1995). Dineserv: a tool for measuring service quality in restaurant. CQ Restaurant Administration Quarterly, 36(2): 56-60. http://dx.doi.org/10.1177/001088049503600226

Taylor, S. A. y Baker, T. L. (1994). An assessment of the relationship between service quality and customer satisfaction in the formation of consumers' purchase intentions. Journal of retailing, 70(2): 163-178. http://dx.doi. org/10.1016/0022-4359(94)90013-2

Taylor, S. A. y Cronin Jr, J. J. (1994). Modeling patient satisfaction and service quality. Journal of Health Care Marketing, 14(1): 34-44.

Tsoukatos, E. y G. Rand (2007). Cultural influences on service quality and customer satisfaction: evidence from Greek insurance. Managing Service Quality, 17(4): 467-485. http://dx.doi.org/10.1108/09604520710760571

Westbrook, R. (1980). Intrapersonal affective influences on consumer satisfaction with products. Journal of Consumer Research, 7(1): 49-54. http://dx.doi.org/10.1086/208792

Wheaton, B., Muthén, B., Alwin, D.F. y Summers, G.F. (1977). Assessing reliability and stability in panel models. In Heise, D.R. [Ed.] Sociological methodology. San Francisco: Jossey-Bass: 84-136.

Woodside, A.G., Frey, L.L. y Daly, R.T. (1989). Linking service quality, customer satisfaction. Journal of Health Care Marketing, December: 5-17.

Van Dyke, T. P., Kappelman, L. A. y Prybutok, V. R. (1997). Measuring information systems service quality: concerns on the use of the SERVQUAL questionnaire. MIS Quarterly, 21(2): 195-208. http://dx.doi.org/10.2307/249419

Vignolo, J., Vacarezza, M., Álvarez, C. y Sosa, A. (2011). Niveles de atención, de prevención y atención primaria de la salud. Archivos de Medicina Interna, 33(1): 7-11.

Zeithaml, V. A., Berry L.L. y Parasuraman, A. (1996). The Behavioral Consequences of Service Quality. Journal of Marketing, 60(2): 31-46. http://dx.doi.org/10.2307/1251929 